Tuning the work function of stepped metal surfaces by adsorption of organic molecules

This content has been downloaded from IOPscience. Please scroll down to see the full text.

2017 J. Phys.: Condens. Matter 29204001

(http://iopscience.iop.org/0953-8984/29/20/204001)

View the table of contents for this issue, or go to the journal homepage for more

Download details:

IP Address: 219.230.111.17

This content was downloaded on 13/04/2017 at 13:25

Please note that terms and conditions apply. 


\title{
Tuning the work function of stepped metal surfaces by adsorption of organic molecules
}

\author{
Yingda Jiang ${ }^{1,4}$, Jingtai $\mathrm{Li}^{1,4}$, Guirong Su${ }^{1}$, Nicola Ferri ${ }^{2}$, Wei Liu ${ }^{1}$ \\ and Alexandre Tkatchenko ${ }^{3}$
}

\footnotetext{
${ }^{1}$ Nano Structural Materials Center, School of Materials Science and Engineering, Nanjing University of Science and Technology, Nanjing 210094, Jiangsu, People's Republic of China

2 Fritz-Haber-Institut der Max-Planck-Gesellschaft, Faradayweg 4-6, D-14195, Berlin, Germany

${ }^{3}$ Physics and Materials Science Research Unit, University of Luxembourg, L-1511 Luxembourg

E-mail: weiliu@njust.edu.cn and alexandre.tkatchenko@uni.lu
}

Received 10 January 2017, revised 5 March 2017

Accepted for publication 27 March 2017

Published 13 April 2017

\begin{abstract}
Understanding the binding mechanisms for aromatic molecules on transition-metal surfaces, especially with defects such as vacancies, steps and kinks, is a major challenge in designing functional interfaces for organic devices. One important parameter in the performance of organic/inorganic devices is the barrier of charge carrier injection. In the case of a metallic electrode, tuning the electronic interface potential or the work function for electronic level alignment is crucial. Here, we use density-functional theory (DFT) calculations with van der Waals (vdW) interactions treated with both screened pairwise (vdW $\left.{ }^{\text {surf }}\right)$ and many-body dispersion (MBD) methods, to systematically study the interactions of benzene with a variety of stepped surfaces. Our calculations confirm the physisorptive character of $\operatorname{Ag}(211), \operatorname{Ag}(533), \operatorname{Ag}(322), \operatorname{Ag}(755)$ and $\operatorname{Ag}(544)$ surfaces upon the adsorption of benzene. The MBD effects reduce the adsorption energies by about $0.15 \mathrm{eV}$ per molecule compared to the results from the DFT $+v_{d W} W^{\text {surf }}$ method. In addition, we find that the higher the step density, the larger the reduction of the work function upon the adsorption of benzene. We also study the effect of vdW interactions on the electronic structure using a fully selfconsistent implementation of the vdW ${ }^{\text {surf }}$ method in the Kohn-Sham DFT framework. We find that the self-consistent vdW ${ }^{\text {surf }}$ effects increase the work function due to the lowered Fermi level and the increased vacuum level. As a result, the benzene/Ag(211) system has the lowest work function $(3.67 \mathrm{eV})$ among the five adsorption systems, significantly smaller than the work function of the clean $\mathrm{Ag}\left(\begin{array}{lll}1 & 1 & 1\end{array}\right)$ surface $(4.74 \mathrm{eV})$. Our results provide important insights into the stability and electronic properties of molecules adsorbed on stepped metal surfaces, which could help in designing more appropriate interfaces with low work functions for electron transfer.
\end{abstract}

Keywords: stepped surfaces, adsorption, work function, self-consistent vdW ${ }^{\text {surf }}$ effects

S. Supplementary material for this article is available online

(Some figures may appear in colour only in the online journal)

\footnotetext{
${ }^{4} \mathrm{YJ}$ and JL contributed equally to this work
} 


\section{Introduction}

Interfaces between organic molecules and metal surfaces play an increasingly important role in numerous organic (opto)electronic devices [1-3]. The performance of efficient devices is often determined by the adsorption and interactions of such hybrid inorganic-organic systems (HIOS). In most cases, close-packed metal surfaces were extensively studied due to their high stability and easy processability [4-7]. However, vacancies, kinks, and steps are inevitable in actual devices, and they often dictate the adsorption dynamics and determine the activity of catalysts [8-14]. Therefore, modeling electronic properties of molecules interacting with non-pristine surfaces is crucial for understanding the relevant properties of realistic HIOS. Notably, stepped surfaces, especially step edge sites, have been proposed to be more suitable than flat surfaces for building ordered two-dimensional molecular structures at low coverage [15-17].

The electron transport properties in HIOS have attracted considerable interest in the field of nanoscale electronics [18]. One important parameter governing electron transport at the metal-organic interface is the work function. Low work function interfaces are appropriate for electron transport $[19,20]$. The work functions of surfaces can be tuned by molecular adsorption and coverage, with van der Waals (vdW) interactions playing a prominent role in the properties of interfaces [21-24]. For example, our recent study found that the adsorption can decrease the work function of the clean $\mathrm{Cu}(111)$ surface by $0.85,0.71$ and $0.74 \mathrm{eV}$ for olympicene, its radical, and its ketone, respectively [23]. In addition, Bauert et al [24] reported that work functions decrease linearly with increasing coverage. The studies above included the vdW force in the calculations, but neglected the pronounced contributions of many-body electronic correlations, which arise from the collective electronic fluctuations in the combined molecule/ surface system [25]. The vdW interactions have been shown to strongly affect the stability and structure of hybrid systems [26-30]. However, the most widely employed vdW-inclusive methods simply add a correction to the density-functional theory (DFT) total energy in the form of a non-local interaction energy [31, 32]. On the other hand, a self-consistent (SC) interatomic vdW density functional adds the electronic $\mathrm{vdW}$ potential to the exchange-correlation potential to form the total Kohn-Sham effective potential [33]. Early studies revealed that a SC treatment of both the vdW-DF functional and density-dependent dispersion correction lead to tiny modifications of the structure and stability of molecular dimers and crystals [34-37]. However, Ferri et al [33] recently reported that SC vdW effects can affect work functions for metal substrates, particularly for $\mathrm{Rh}$ and $\mathrm{Ag}$, and at organic/ metal interfaces. As such, their findings are also strongly suggestive of the potential importance of long-range vdW effects in properties beyond the electronic ground state. However, they only studied the (111) close-packed metal surfaces.

In this work, we systematically study the adsorption of benzene on stepped (211), (533), (322), (755) and (544) surfaces of $\mathrm{Ag}$, due to the frequent use of $\mathrm{Ag}$ as an electrode. We use DFT calculations with vdW interactions treated with both screened pairwise (vdW ${ }^{\text {surf }}$ ) [21] and many-body dispersion (MBD) $[25,38]$ methods. Our calculations show that the benzene is weakly adsorbed on the five surfaces with a small amount of charge transfer, which indicates a physisorptive character. Thus, the adsorption energies are close to that of benzene/Ag(111). On the other hand, the adsorption of benzene induces a linear reduction versus increasing step density in the work function for all five surfaces. Consequently, the work function of $\operatorname{Ag}(211)$ decreases the most upon benzene adsorption (by $0.92 \mathrm{eV}$ ) in comparison with the clean $\operatorname{Ag}(211)$ surface when treating vdW interactions fully self-consistently. Therefore, low work functions can be obtained by tuning the work functions of metal surfaces via molecular adsorption and steps.

\section{Computational details}

We performed DFT calculations using the semi-local Perdew-Burke-Ernzerhof (PBE) exchange-correlation functional [39]. Several methods of increasing complexity are used in this work to model structure, stability, and electronic properties of stepped Ag surfaces. To guide the reader, here we provide a succinct description of the different methods employed throughout this work:

(i) DFT $+v_{d W}^{\text {surf: }}$ The DFT $+v$ WW $^{\text {surf }}$ method [21] combines the effective pairwise atom-atom approximation with the electrodynamic screening of vdW interactions employing the Lifshitz-Zaremba-Kohn theory [40] for the dielectric screening within the metal surface. This approach adds the dispersion energy to the DFT total energy given as a sum of $-C_{6}^{a b} R_{a b}^{-6}$ terms, where $R_{a b}$ is the distance between atoms $a$ and $b$. The collective screening of the substrate electrons captured via the $\mathrm{vdW}^{\text {surf }}$ method, are in the determination of the $C_{6}$ coefficients and vdW radii (vdW parameters), going beyond the interatomic pairwise description effectively.

(ii) DFT + MBD: Different from the vdW ${ }^{\text {surf }}$ method, the many-body dispersion (MBD) method [25, 38] computes the long-range correlation energy through the coupled harmonic oscillator model Hamiltonian, and treats dipolar vdW interactions to all orders in perturbation theory beyond the vdW ${ }^{\text {surf }}$ method. Moreover, the MBD method avoids the explicit use of single-electron orbitals, allowing for a favorable $N^{3}$ scaling ( $N$ is the number of atoms) and a negligible computational cost. This method has been demonstrated to perform very well for supramolecular systems, molecular crystals, nanoclusters, and layered nanostructures [25, 41-45].

(iii) $\mathrm{DFT}+\mathrm{sc}-\mathrm{vdW}^{\text {surf: }}$ We also used the self-consistent $\mathrm{vdW}^{\text {surf }}$ (sc-vdW $\mathrm{w}^{\text {surf }}$ ) method [33] to examine the effects of $\mathrm{vdW}$ interactions on the work function of benzene on Ag stepped surfaces. In the sc-vdW ${ }^{\text {surf }}$ method the electronic vdW potential is added to the exchange-correlation potential to form the total Kohn-Sham effective potential, modifying the single-particle eigenfunctions and eigenvalues resulting from a SC solution of the electronic Kohn-Sham equations. 
All DFT calculations in this work have been performed using the 'tight' computational settings in the FHI-aims all-electron code [46, 47], applying a scaled zeroth-order regular approximation (ZORA) for treating relativistic effects. We set the following thresholds for the convergence criteria: $10^{-2} \mathrm{eV} \AA^{-1}$ for the final forces in all structural relaxations, $10^{-5}$ electrons for the electron density, and $10^{-5} \mathrm{eV}$ for the total energy of the system.

Here, we focus on five stepped surfaces with the same Miller indices of $\operatorname{Ag}(n+1, n-1, n-1)$, with $n=3,4,5$, 6 and 9. Note that $n$ denotes the number of atom rows on the (111) terraces that are separated by monoatomic (100) steps. Thus, the above stepped surfaces can also be noted as $\operatorname{Ag}\left[n\left(\begin{array}{lll}1 & 1 & 1\end{array}\right) \times\left(\begin{array}{lll}1 & 0 & 0\end{array}\right)\right]$ [48]. For each surface, the step density, $N$, is defined as $N=1 /[D(n-1 / 2)]$ (step sites per unit length) [49], where $D$ is the atomic diameter of $\operatorname{Ag}(2.88 \AA)$. For the adsorption of benzene, the $\operatorname{Ag}(211),(533),(322),(755)$ and $(544)$ surfaces were modeled by periodic $(1 \times 3)$ unit cells, containing 15, 20, 26, 30 and 44 atomic layers separated by at least $100 \AA$ of vacuum. For geometry optimizations, the molecule and the 6, 8, 10,12 and 19 uppermost metal layers were fully relaxed, while the metal atoms of the bottommost $9,12,16,18$ and 25 layers were constrained at their bulk positions. These computational settings guarantee a convergence in the adsorption energies and work functions better than 20 and $30 \mathrm{meV}$, respectively (see tables $\mathrm{S} 1-\mathrm{S} 3$ ).

\section{Results and discussion}

\subsection{Adsorption energies and equilibrium geometries}

We first determined the most preferable geometries for benzene on $\operatorname{Ag}(211),(533),(322),(755)$ and (544) surfaces at step and terrace sites ('top', 'bridge', 'hcp-hollow' and 'fcc-hollow'), and two orientations of $\mathrm{C}-\mathrm{C}$ bond being rotated with respect to the neighboring metal-metal bond $\left(0^{\circ}\right.$ and $\left.30^{\circ}\right)$ with exhaustive methods (see figure $\mathrm{S} 1$ ). We used the PBE + $\mathrm{vdW}^{\text {surf }}$ method for geometry relaxation and further employed the MBD method for single-point energy calculations at the $\mathrm{PBE}+\mathrm{vdW}$ surf geometry. The adsorption energies $\left(E_{\mathrm{ads}}\right)$ of benzene on Ag stepped surfaces were determined by,

$$
E_{\mathrm{ads}}=E_{\mathrm{sys}}-E_{\mathrm{M}}-E_{\mathrm{Bz}}
$$

where $E_{\mathrm{sys}}, E_{\mathrm{M}}$, and $E_{\mathrm{Bz}}$ denotes the total energy of the adsorption system, the relaxed bare metal slab, and the relaxed gas-phase benzene, respectively.

The most stable adsorption structures are found at the bridge site on step edges (see figure 1). As shown in table 1, the largest adsorption energy of benzene is on $\operatorname{Ag}(322)$ with both $\mathrm{PBE}+\mathrm{vdW}^{\text {surf }}(-0.85 \mathrm{eV})$ and $\mathrm{PBE}+\mathrm{MBD}$ $(-0.70 \mathrm{eV})$ methods. Oppositely, benzene/Ag(544) and benzene/Ag(211) have the smallest adsorption energies with $\mathrm{PBE}+\mathrm{vdW}^{\text {surf }}(-0.76 \mathrm{eV})$ and $\mathrm{PBE}+\mathrm{MBD}(-0.62 \mathrm{eV})$. The PBE + MBD adsorption energies are consistently smaller than those of $\mathrm{PBE}+\mathrm{vdW}^{\text {surf }}$. The reduction in binding at the PBE + MBD level when compared with PBE $+v_{d W}^{\text {surf }}$ stems from significant interface polarization redistribution due to many-body correlation effects [45]. Interestingly, the many-body effects yield very similar contributions in the binding energies of the different stepped surfaces. Actually, the largest difference in binding energies is only $0.08 \mathrm{eV}$ per molecule with both $\mathrm{PBE}+\mathrm{MBD}$ and $\mathrm{PBE}+\mathrm{vdW}^{\text {surf }}$ and is found between benzene/ $\operatorname{Ag}(322)$ and benzene $/ \operatorname{Ag}(211)$. To understand the almost identical adsorption energies, we decompose the total adsorption energies into local and non-local contributions. We find that the contributions either from $\mathrm{PBE}$ or MBD all have small differences among the five systems (less than $0.07 \mathrm{eV}$ per molecule). The smaller adsorption energies also indicate the physisorptive character of $\mathrm{Ag}$ stepped surfaces. Besides energetics, the nature of bonding in the five systems can also be evidenced by their adsorption geometries. As shown in figure 1, the distances are larger than $2.79 \AA$ between the center of mass of the molecule and the step edge of the surface. Figure 1 also illustrates that benzene tends to be parallel to the terrace with increasing terrace width. The adsorption energy of benzene/ $\operatorname{Ag}(544)$ is $-0.76 \mathrm{eV}$ from $\mathrm{PBE}+\mathrm{vdW}^{\text {surf }}$, which is close to that of benzene/Ag( 1111$)$ $(-0.79 \mathrm{eV})$ [50]. Therefore, the adsorption of adsorbates on the series of $\operatorname{Ag}(n+1, n-1, n-1)$ stepped surfaces would be similar to the (111) surface when $n$ is sufficiently large.

\subsection{Electronic properties of the adsorption systems}

Besides adsorption structures and energetics, we also computed projected densities of states on the molecule (MODOS) for benzene on Ag stepped surfaces, from which the physisorptive character of the systems can be clearly seen. As shown in figure 2, the adsorption breaks the degeneracy of HOMO and HOMO- 1 orbitals of the adsorbed benzene, due to the perturbation by the metal substrates. The analysis of the occupation of projected orbitals indicates that for $\operatorname{Ag}(211)$, (533), (322), (755) and (544) surfaces there are more than 1.888 electrons in the HOMO orbital. Meanwhile, the occupation number of the LUMO state is less than 0.039 electrons. The electronic occupation number indicates a small amount of charge transfer upon the adsorption of benzene and further demonstrates the physisorptive character of the five surfaces. We also compared the MODOS of the five stepped systems with benzene/ $\operatorname{Ag}(111)$ [50]. Figure 2(f) shows that the HOMO and HOMO-1 orbitals of the adsorbed benzene on $\operatorname{Ag}(111)$ remain degenerate, which indicates that the stepped surfaces are more reactive than the $\operatorname{Ag}(111)$ surface.

The redistribution of charge upon adsorption is most sensitively reflected in a change of the surface dipole, and hence in the work function. Therefore, we computed the change in the work function of the stepped surfaces due to the adsorption of benzene (figure 3), compared with the benzene/Ag(111) system. We find that work functions of the five clean stepped surfaces are close to each other (with less than $0.16 \mathrm{eV}$ difference), and are all smaller than the one of the $\operatorname{Ag}(111)$ surface (4.44 eV) [33].

Figure 3 also shows that the adsorption of benzene on the five stepped surfaces leads to a reduction in the work function compared to the clean surfaces. More importantly, when increasing step density from $0.04(n=9)$ to $0.14(n=3) \AA^{-1}$ (surface coverage from 0.55 to $1.64 \mathrm{~nm}^{-2}$ ) we find a monotonous decrease 
(a)

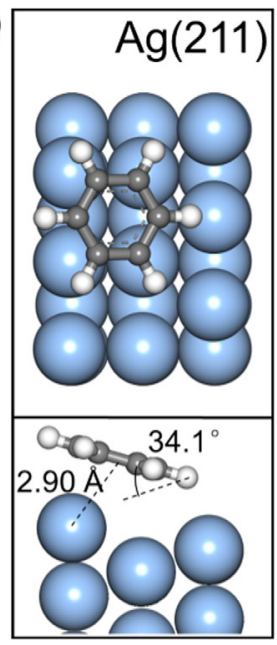

(b)

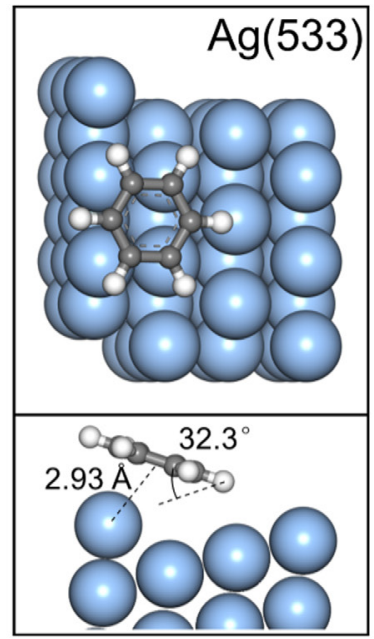

(c)

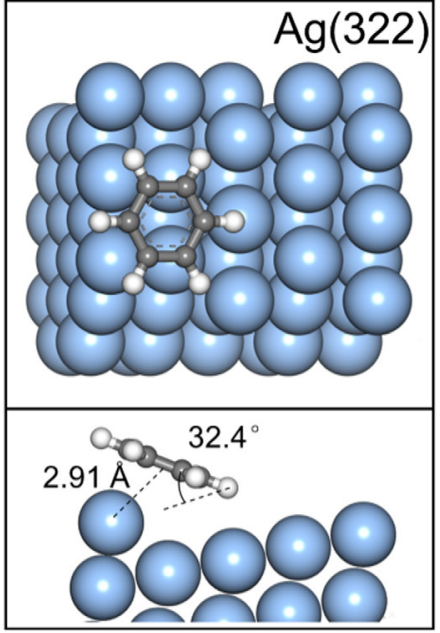

(d)
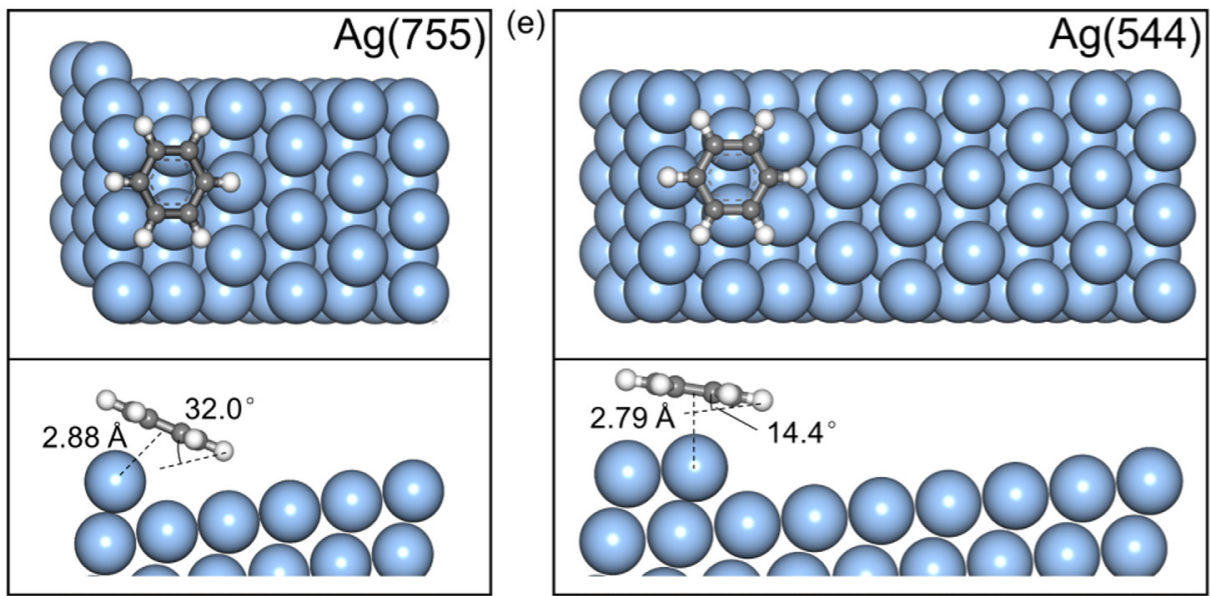

Figure 1. Top views and side views of a benzene molecule adsorbed on (a) $\operatorname{Ag}(211$ ), (b) $\operatorname{Ag}(533),(c) \operatorname{Ag}(322),(d) \operatorname{Ag}(755)$ and (e) $\operatorname{Ag}(544)$ stepped surfaces. Blue, grey and white denote silver, carbon and hydrogen atoms, respectively. The distance between the geometric center of the molecule and the step edge atoms, and the angle between the molecule and the plane of terrace are also shown in the figure.

Table 1. Computed binding energies and adsorption structures for benzene on stepped surfaces of $\mathrm{Ag}$ at the most preferable sites. The PBE and MBD values are decomposed from the PBE + MBD adsorption energies. $d$ is distance between the center of mass of the molecule and the step edge of the surface. $\theta$ is the angle between the molecule and the plane of terrace.

\begin{tabular}{|c|c|c|c|c|c|c|}
\hline \multirow[b]{2}{*}{ Systems } & \multicolumn{4}{|c|}{$E_{\text {ads }}(\mathrm{eV})$} & \multirow[b]{2}{*}{$d(\AA)$} & \multirow[b]{2}{*}{$\theta\left(^{\circ}\right)$} \\
\hline & $\mathrm{PBE}+\mathrm{vdW} \mathrm{W}^{\text {surf }}$ & $\mathrm{PBE}+\mathrm{MBD}$ & PBE & MBD & & \\
\hline Benzene/Ag(211) & -0.77 & -0.62 & -0.01 & -0.61 & 2.90 & 34.1 \\
\hline Benzene/Ag(533) & -0.81 & -0.65 & -0.04 & -0.61 & 2.93 & 32.3 \\
\hline Benzene/Ag(322) & -0.85 & -0.70 & -0.05 & -0.65 & 2.91 & 32.4 \\
\hline Benzene/Ag(755) & -0.83 & -0.68 & -0.04 & -0.64 & 2.88 & 32.0 \\
\hline Benzene/Ag(544) & -0.76 & -0.63 & -0.05 & -0.58 & 2.79 & 14.4 \\
\hline
\end{tabular}

of work function from 3.92 to $3.49 \mathrm{eV}$ upon the adsorption of benzene. This leads to the reduction of work function values in the range of $0.43-0.71 \mathrm{eV}$ with respect to the clean surfaces. In general, the molecular adsorption changes the work function of the bare surface by generating an interface dipole. To understand the decrease in the work function values, we decompose the total dipole into the dipole at the interface, called bond dipole, and the molecular dipole perpendicular to the surface [51]. As shown in table 2 , the reduction of work function is mainly due to the bond dipole, which is an effect of the redistribution of electron density. For weakly bound benzene, the bond dipole is predominantly attributed to the effect of Pauli repulsion, or 'push-back' effect [52]. Due to the physisorptive character of benzene on the studied surfaces, there is negligible deformation of the molecular structure which can induce a molecular dipole that would also contribute to the change in the interface work function. Note that, increasing the surface coverage from 0.25 monolayers (ML) to $1 \mathrm{ML}$ can also lead to a rapid reduction in work functions [24], which demonstrates again that the work function can be tailored by surface coverage. 

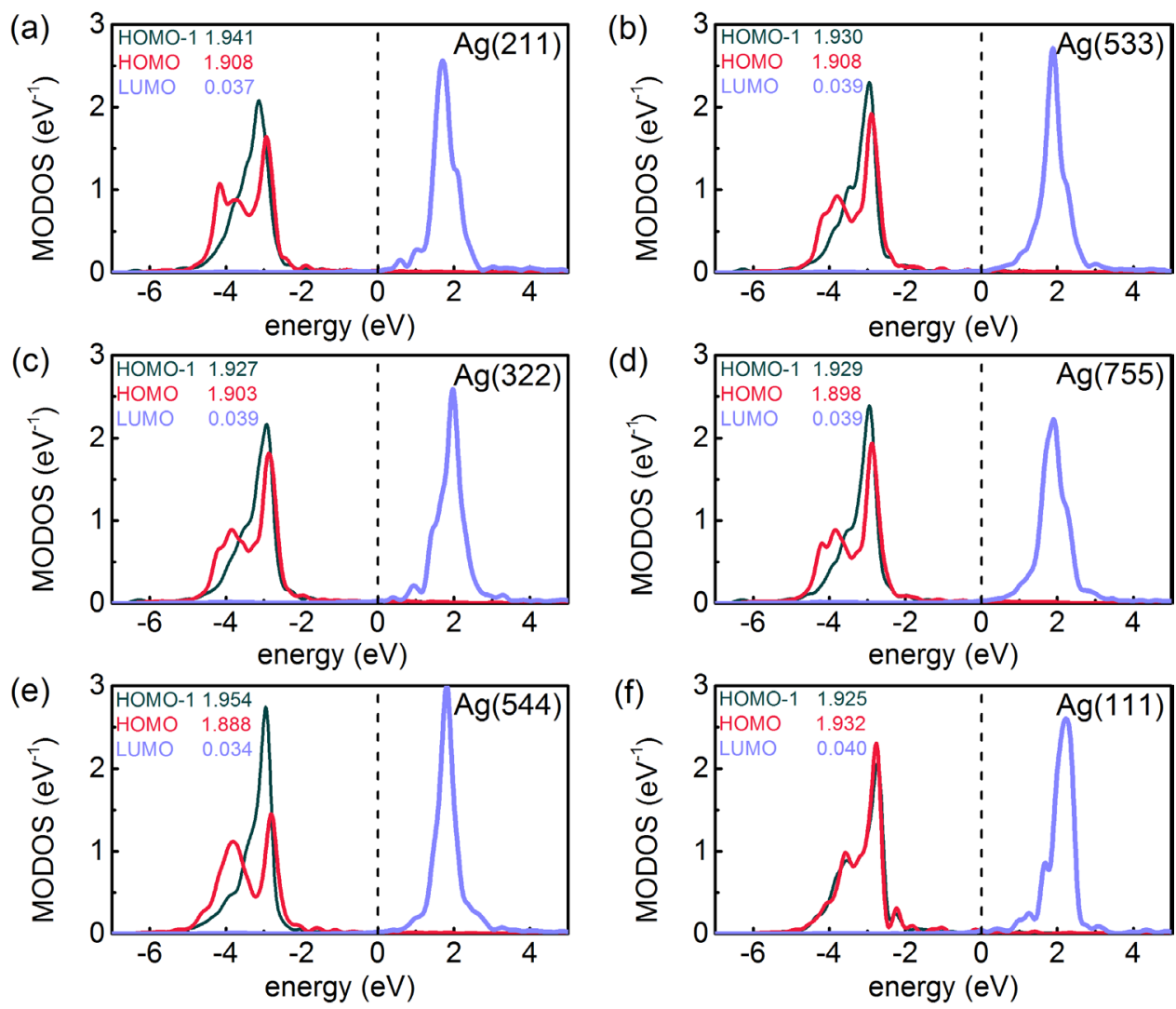

Figure 2. Density of states projected on the free benzene molecule HOMO-1, HOMO and LUMO orbitals for benzene on (a) $\mathrm{Ag}(211)$,

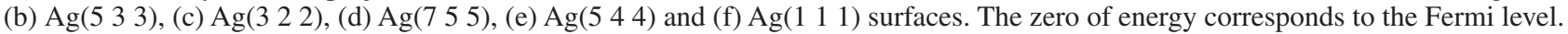

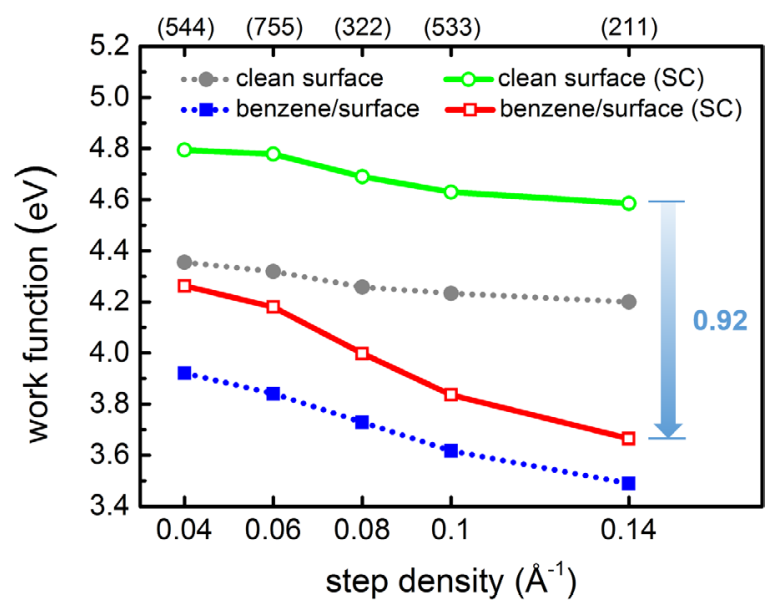

Figure 3. Work functions for clean Ag surfaces and benzene/Ag adsorption systems versus step density, calculated with $\mathrm{PBE}+\mathrm{vdW} \mathrm{Wurf}^{\text {sundicated by dotted lines) and } \mathrm{PBE}+\mathrm{sc}-\mathrm{vdW} \text { surf }}$ (solid lines).

Finally, we utilized the sc-vdW ${ }^{\text {surf }}$ method to examine the effects of electronic self-consistency in vdW interactions on the work function of benzene on Ag stepped surfaces. For the five clean surfaces the work functions computed with the $\mathrm{PBE}+\mathrm{sc}-\mathrm{vdW}{ }^{\text {surf }}$ method are $4.59-4.74 \mathrm{eV}$, being at least $0.39 \mathrm{eV}$ larger than those of PBE $+\mathrm{vdW}^{\text {surf }}$. Since the $\mathrm{vdW}$ radius of the $\mathrm{Ag}$ atom $(2.57 \AA)$ is smaller than the interatomic distance of $\mathrm{Ag}$ ( $2.88 \AA$ ), self-consistency yields to a net accumulation of electron density in the interstitial and vacuum regions coupled with a depletion within the metal layers.
Consequently, the combination of the lowered Fermi level due to the attractive Coulombic interaction between the metal layers and the interstitial region, and an increased vacuum level, induced by a larger surface dipole due to the extension of the electron density above the surface, ultimately increases the work function of $\operatorname{Ag}(111)$ surface from 4.44 to $4.74 \mathrm{eV}$ [33] (see table S4).

Note that, the adsorption of benzene leads to a significant reduction $(0.53-0.92 \mathrm{eV})$ in the work function of the surface with the PBE + sc-vdW ${ }^{\text {surf }}$ method, larger than that with $\mathrm{PBE}+\mathrm{vdW}^{\text {surf }}$. The $\operatorname{Ag}(211)$ surface has the lowest work function upon the adsorption of benzene among these five surfaces. The self-consistent work function of benzene/ $\operatorname{Ag}(211)$, which has the largest surface coverage (1.64 $\mathrm{nm}^{-2}$ ), is $0.59 \mathrm{eV}$ lower than that of benzene/ $\operatorname{Ag}(544)$ with the smallest surface coverage $\left(0.55 \mathrm{~nm}^{-2}\right)$. However, the work function of $\operatorname{Ag}(211)$ is $0.20 \mathrm{eV}$ lower than that of $\operatorname{Ag}(544)$. Therefore, our calculations clearly demonstrate that the reduction of the work function stems from two aspects: both the increased surface coverage and the increased step density of metal surfaces.

The large reduction in the work function is due to the modification of the interface dipole of the surfaces. In this regard, the SC effects considerably modify the Fermi levels of the five systems. In contrast, the computed Fermi levels of the non-selfconsistent method are close to each others. This leads to the different slopes of self-consistent work functions, in comparison to those from the PBE $+\mathrm{vdW}^{\text {surf }}$ method (see table S5). In analogy to the results computed by $\mathrm{PBE}+\mathrm{vdW}^{\text {surf }}$, 
Table 2. Work function change $\Delta \phi^{\text {Total }}$ (in eV) for benzene/Ag systems computed with $\mathrm{PBE}+\mathrm{vdW}$ surf and PBE $+\mathrm{sc}-\mathrm{vdW}$ surf $\Delta \phi^{\text {Total }}=-\left(\mathrm{WF}_{\text {system }}-\mathrm{WF}_{\mathrm{M}}\right)$, where $\mathrm{WF}_{\text {system }}$ and $\mathrm{WF}_{\mathrm{M}}$ denote the work function of the adsorption system and the clean metal surface, respectively. $\Delta \phi^{\text {Total }}$ is divided into two components: one from the bond dipole $\Delta \phi^{\text {Bond }}$ and the other from the molecular dipole $\Delta \phi^{\text {Mol }}$.

\begin{tabular}{|c|c|c|c|c|c|c|}
\hline \multirow[b]{2}{*}{ Systems } & \multicolumn{3}{|c|}{$\mathrm{PBE}+\mathrm{vdW}$ surf } & \multicolumn{3}{|c|}{$\mathrm{PBE}+$ sc-vdW $\mathrm{Wurf}^{\text {sur }}$} \\
\hline & $\Delta \phi^{\text {Total }}$ & $\Delta \phi^{\text {Bond }}$ & $\Delta \phi^{\mathrm{Mol}}$ & $\Delta \phi^{\text {Total }}$ & $\Delta \phi^{\text {Bond }}$ & $\Delta \phi^{\mathrm{Mol}}$ \\
\hline Benzene/Ag(211) & 0.710 & 0.708 & 0.002 & 0.920 & 0.918 & 0.002 \\
\hline Benzene/Ag(533) & 0.617 & 0.614 & 0.003 & 0.794 & 0.791 & 0.003 \\
\hline Benzene/Ag(322) & 0.529 & 0.517 & 0.012 & 0.692 & 0.680 & 0.012 \\
\hline Benzene/Ag(7 5 5) & 0.478 & 0.467 & 0.011 & 0.598 & 0.587 & 0.011 \\
\hline Benzene/Ag(544) & 0.434 & 0.434 & 0 & 0.531 & 0.531 & 0 \\
\hline
\end{tabular}

the changes of work function with PBE + sc-vdW ${ }^{\text {surf }}$ method are also due to the bond dipole. However, the values of bond dipole increase significantly with respect to those without vdW self consistency (table 2). We thus conclude that there is a non-trivial connection between electrostatics and long-range electron correlation effects, which affects the computed work functions.

\section{Conclusion}

We have used the screened vdW and many-body dispersion methods to systematically study benzene adsorbed on the stepped surfaces of Ag. We find that the MBD effects play a prominent role for all the systems studied, reducing the binding energies by about $0.15 \mathrm{eV}$ per molecule with respect to the results from the DFT $+v_{d W}{ }^{\text {surf }}$ method. Our calculations also show that work functions can be tailored by the surface coverage and step density of metal surfaces. The higher the step density, the larger the reduction of the work function. We further examine the effects of $\mathrm{vdW}$ interactions on the work function and find that the sc-vdW ${ }^{\text {surf }}$ effects can lead to larger reduction of the work function in comparison to DFT + vdW ${ }^{\text {surf }}$. As a result, the $\operatorname{Ag}(211)$ stepped surface with the highest step density would be the most promising candidate to design electronic devices, due to its lowest work function upon the adsorption of benzene.

\section{Acknowledgments}

WL acknowledges support from the NSF of China (21403113), the NSF of Jiangsu Province (BK20150035), and the Foundation of Jiangsu Specially-Appointed Professor. We are grateful for support from Jiangsu Key Laboratory of Advanced Micronano Materials and Technology. AT thanks the European Research Council for financial support (ERC-StG VDW-CMAT).

\section{References}

[1] Koch N, Ueno N and Wee A T S 2013 The Molecule-Metal Interface (New York: Wiley)

[2] Al Ma'Mari F et al 2015 Nature $\mathbf{5 2 4} 69$

[3] Tkatchenko A 2015 Adv. Funct. Mater. 252054

[4] Kelkkanen A K, Lundqvist B I and Nørskov J K 2011 Phys. Rev. B 83113401
[5] Lukas S, Vollmer S, Witte G and Wöll C 2001 J. Chem. Phys. 11410123

[6] Tonigold K and Groß A 2010 J. Chem. Phys. 132224701

[7] Berland K, Einstein T and Hyldgaard P 2009 Phys. Rev. B 80155431

[8] Camarillo-Cisneros J, Liu W and Tkatchenko A 2015 Phys. Rev. Lett. 115086101

[9] Vang R T, Honkala K, Dahl S, Vestergaard E K, Schnadt J, Lægsgaard E, Clausen B S, Nørskov J K and Besenbacher F 2005 Nat. Mater. 4160

[10] Rostrup-Nielsen J and Nørskov J K 2006 Top. Catal. 4045

[11] Nørskov J K, Bligaard T, Hvolbæk B, Abild-Pedersen F, Chorkendorff I and Christensen C H 2008 Chem. Soc. Rev. 372163

[12] Hendriksen B L et al 2010 Nat. Chem. 2730

[13] Zhu Q, Saidi W A and Yang J C 2016 J. Phys. Chem. Lett. 72530

[14] Hussain A, Ferré D C, Gracia J, Nieuwenhuys B and Niemantsverdriet J 2009 Surf. Sci. $\mathbf{6 0 3} 2734$

[15] Zambelli T, Jiang P, Lagoute J, Grillo S, Gauthier S, Gourdon A and Joachim C 2002 Phys. Rev. B 66075410

[16] Pascual J I, Jackiw J J, Kelly K F, Conrad H, Rust H P and Weiss P S 2000 Phys. Rev. B 6212632

[17] Peköz R, Johnston K and Donadio D 2012 J. Phys. Chem. C 11620409

[18] Neaton J B, Hybertsen M S and Louie S G 2006 Phys. Rev. Lett. 97216405

[19] Cahen D and Kahn A 2003 Adv. Mater. 15271

[20] Zoppi L, Martin-Samos L and Baldridge K K 2014 Acc. Chem. Res. 473310

[21] Ruiz V G, Liu W, Zojer E, Scheffler M and Tkatchenko A 2012 Phys. Rev. Lett. 108146103

[22] Bürker C, Ferri N, Tkatchenko A, Gerlach A, Niederhausen J, Hosokai T, Duhm S, Zegenhagen J, Koch N and Schreiber F 2013 Phys. Rev. B 87165443

[23] Liu W, Schuler B, Xu Y, Moll N, Meyer G, Gross L and Tkatchenko A 2016 J. Phys. Chem. Lett. 71022

[24] Bauert T, Zoppi L, Koller G, Garcia A, Baldridge K K and Ernst K H 2011 J. Phys. Chem. Lett. 22805

[25] Ambrosetti A, Reilly A M, DiStasio R A Jr and Tkatchenko A 2014 J. Chem. Phys. 140 18A508

[26] Reckien W, Eggers M and Bredow T 2014 Beilstein J. Org. Chem. 101775

[27] Liu W, Ruiz V G, Zhang G X, Santra B, Ren X, Scheffler M and Tkatchenko A 2013 New J. Phys. 15053046

[28] Liu W, Filimonov S N, Carrasco J and Tkatchenko A 2013 Nat. Commun. 42569

[29] Liu W, Carrasco J, Santra B, Michaelides A, Scheffler M and Tkatchenko A 2012 Phys. Rev. B 86245405

[30] Ruiz V G, Liu W and Tkatchenko A 2016 Phys. Rev. B 93035118

[31] Grimme S 2006 J. Comput. Chem. 271787

[32] Tkatchenko A and Scheffler M 2009 Phys. Rev. Lett. 102073005 
[33] Ferri N, DiStasio R A Jr, Ambrosetti A, Car R and Tkatchenko A 2015 Phys. Rev. Lett. 114176802

[34] Thonhauser T, Cooper V R, Li S, Puzder A, Hyldgaard P and Langreth D C 2007 Phys. Rev. B 76125112

[35] Langreth D C et al 2009 J. Phys.: Condens. Matter 21084203

[36] Mura M, Gulans A, Thonhauser T and Kantorovich L 2010 Phys. Chem. Chem. Phys. 124759

[37] Brémond É, Golubev N, Steinmann S N and Corminboeuf C 2014 J. Chem. Phys. 140 18A516

[38] Tkatchenko A, DiStasio R A Jr, Car R and Scheffler M 2012 Phys. Rev. Lett. 108236402

[39] Perdew J P, Burke K and Ernzerhof M 1996 Phys. Rev. Lett. 773865

[40] Zaremba E and Kohn W 1976 Phys. Rev. B 132270

[41] Kronik L and Tkatchenko A 2014 Acc. Chem. Res. 473208

[42] Maurer R J, Ruiz V G and Tkatchenko A 2015 J. Chem. Phys. 143102808

[43] Reilly A M and Tkatchenko A 2015 Chem. Sci. 63289
[44] Hansen K, Biegler F, Ramakrishnan R, Pronobis W, Von Lilienfeld O A, Müller K R and Tkatchenko A 2015 J. Phys. Chem. Lett. 62326

[45] Liu W, Maaß F, Willenbockel M, Bronner C, Schulze M, Soubatch S, Tautz F S, Tegeder P and Tkatchenko A 2015 Phys. Rev. Lett. 115036104

[46] Blum V, Gehrke R, Hanke F, Havu P, Havu V, Ren X, Reuter K and Scheffler M 2009 Comput. Phys. Commun. 1802175

[47] Havu V, Blum V, Havu P and Scheffler M 2009 J. Comput. Phys. 2288367

[48] Lang B, Joyner R W and Somorjai G A 1972 Surf. Sci. 30454

[49] Rodes A 1991 PhD Thesis University of Alicante

[50] Jiang Y, Yang S, Li S and Liu W 2016 Sci. Rep. 639529

[51] Rangger G M, Hofmann O T, Romaner L, Heimel G, Bröker B, Blum R P, Johnson R L, Koch N and Zojer E 2009 Phys. Rev. B 79165306

[52] Braun S, Salaneck W R and Fahlman M 2009 Adv. Mater. 211450 